\title{
Implications and Applications of Fermi Scale Quantum Gravity
}

\author{
U.V.S. Seshavatharam ${ }^{1}$ and S. Lakshminarayana ${ }^{2}$ \\ ${ }^{1}$ Honorary faculty, I-SERVE, Survey no-42, Hitech city, Hyderabad-84,Telangana, INDIA \\ 2 Dept. of Nuclear Physics, Andhra University, Visakhapatnam-03,AP, INDIA \\ Emails: seshavatharam.uvs@gmail.com (and) lnsrirama@gmail.com \\ Orcid numbers : 0000-0002-1695-6037 (and) 0000-0002-8923-772X
}

\begin{abstract}
To understand the mystery of final unification, in our earlier publications, we proposed two bold concepts:1) There exist three atomic gravitational constants associated with electroweak, strong and electromagnetic interactions. 2) There exists a strong elementary charge in such a way that its squared ratio with normal elementary charge is close to reciprocal of the strong coupling constant. In this paper we propose that, $(\hbar c)$ can be considered as a compound physical constant associated with proton mass, electron mass and the three atomic gravitational constants. With these ideas, an attempt is made to understand nuclear stability and binding energy. In this new approach, nuclear binding energy can be fitted with four simple terms having one unique energy coefficient with a formula, $B_{A} \cong\left\{(1-0.00189 \sqrt{Z N}) A-A^{1 / 3}-(Z / N)-\left[\left(A_{s}-A\right)^{2} / A_{s}\right]\right\} 10.09 \mathrm{MeV}$ where $A_{s} \cong 2 Z+0.00642 Z^{2}$ is an estimated mean stable mass number. With this new approach, Newtonian gravitational constant can be estimated in a verifiable approach with a model relation of the form, $\left(\frac{m_{p}}{m_{e}}\right) \cong\left\{\frac{4 \pi^{2}}{\alpha} \sqrt{\frac{\hbar c}{G_{N} m_{p}^{2}}}\right\}^{1 / 7}$
\end{abstract} where $\alpha$ is the Fine structure constant. Estimated $\left(m_{p} / m_{e}\right) \cong 1836.266122$ and is 62 ppm higher than the CODATA recommended $\left(m_{p} / m_{e}\right)$. It needs further investigation. Proceeding further, an attempt is made to fit the recommended quark masses.

Keywords: Four gravitational constants; Compound reduced Planck's constant; Nuclear elementary charge; Strong coupling constant; Nuclear stability and binding energy; Quark masses;

\section{Introduction to large gravitational coupling constants}

To understand the strong interaction, from 1974 to 1993, Tennakone, De Sabbata, Gasperini, Abdus Salam, Sivaram and K.P. Sinha [1-4] tried to introduce a large nuclear gravitational coupling constant. To understand weak interactions, in 2013, Roberto Onofrio [5] introduced a large electroweak gravitational coupling constant. In our 2011 and 2012 papers $[6,7]$ and recently published papers [8-16], we introduced a very large electromagnetic gravitational coupling constant. In this context, we appeal that,

1) Success of any unified model depends on its ability to involve gravity in microscopic models.

2) Full-fledged implementation of gravity in microscopic physics must be able to: a) Estimate the ground state elementary particle rest masses of the three atomic interactions.

b) Estimate the coupling constants of the three atomic interactions.

c) Estimate the range of all interactions.

d) Estimate the Newtonian gravitational constant.

3) As the root is unclear and unknown, to make it success or to have a full-fledged implementation, one may be forced to consider a new path that may be out-of-scope of the currently believed unsuccessful unified physics.

4) In our approach,

a) We assign a different gravitational constant for each basic interaction.

b) We consider proton and electron as the two characteristic building blocks of the four basic interactions. 
c) Finally, by eliminating the three atomic gravitational constants, we develop a characteristic relation for estimating the Newtonian gravitational constant.

d) During this journey, without considering arbitrary numbers or coefficients, we come across many strange and interesting relations for estimating other atomic and nuclear coupling constants.

5) We strongly believe that, with further study, research and synthesizing the noticed relations in a systematic approach, actual essence of final unification can be understood.

In this paper, by considering the three atomic gravitational coupling constants, we review our recently published four REFERENCE relations [8] with reference to $(\hbar c)$ and tried to infer the proposed four term semi empirical mass formula. In section 7 , we tried to fit quark masses. Proceeding further, without considering the nature of forces, we tried to fit the range of weak, strong, electromagnetic and gravitational interactions with a common formula. See point-10 of Discussion.

\section{History and current status of nuclear binding energy scheme}

With respect to nuclear binding energy and semi empirical mass formula (SEMF), the inverse problem framework [17], allows to infer the underlying model parameters from experimental observation, rather than to predict the observations from the model parameters. Recently, the ground-state properties of nuclei with $Z=8$ to 120 from the proton drip line to the neutron drip line have been investigated using the spherical relativistic continuum Hartree-Bogoliubov (RCHB) theory [18] with the relativistic density functional PC-PK1. In this context, in our recently published paper [8], we emphasized the fact that, physics and mathematics associated with fixing of the energy coefficients of SEMF are neither connected with residual strong nuclear force nor connected with strong coupling constant. N. Ghahramany and team members are constantly working on exploring the secrets of nuclear binding energy and magic numbers in terms of quarks $[19,20]$. Very interesting point of their study is that - nuclear binding energy can be understood with two or three terms having single energy coefficient of the order of $10 \mathrm{MeV}$.

\section{Motivating concepts/Basic Ideas/Assumptions}

Even though celestial objects that show gravity are confirmed to be made up of so many atoms, so far scientists could not find any relation in between gravity and the atomic interactions. It clearly indicates that, there is something wrong in our notion of understanding or developing the unified physical concepts. To develop new and workable ideas, we emphasize that,

1) Whether particle's massive nature is due to electromagnetism or gravity or weak interaction or strong interaction or cosmic dust or something else, is unclear.

2) Without understanding the massive nature, it is not reasonable to classify the field created by any elementary particle.

3) All the four interactions seem to be associated with $(\hbar)$.

4) Nobody knows the mystery of $(\hbar)$ which seems to be a basic measure of angular momentum.

5) Nobody knows the mystery of existence, stability and behavior of 'proton' or 'electron'.

6) 'Mass' is a basic property of space-time curvature and basic ingredient of angular momentum.

7) Atoms are mainly characterized by protons and electrons.

8) 'Free neutron' is an unstable particle.

Based on the above points, we propose the following new and workable concepts.

Bold idea-1: The four basic interactions can be allowed to have four different gravitational constants.

Bold idea-2: There exists a strong elementary charge in such a way that its squared ratio with normal elementary charge is close to inverse of the strong coupling constant.

Bold idea-3: $(\hbar c)$ can be considered as a compound physical constant associated with proton mass, electron mass and the three atomic gravitational constants.

With the proposed first two [8-16] concepts, it seems possible to have many applications out of which nuclear stability and binding energy can be understood very easily. In addition to that, Newtonian 
gravitational constant can be estimated in a verifiable approach. We appeal that, by considering the third bold idea, it may be possible to understand the combined role of the four gravitational constants in understanding the vector and tensor nature of fundamental forces and their interaction range.

\section{Quantitative relations}

(1) Let, Newtonian gravitational constant $=G_{N}$ Electromagnetic gravitational constant $=G_{e}$ Nuclear gravitational constant $=G_{s}$ Weak gravitational constant $=G_{w}$

$$
\begin{aligned}
& G_{e} \cong 2.374335 \times 10^{37} \mathrm{~m}^{3} \mathrm{~kg}^{-1} \mathrm{sec}^{-2} \\
& G_{s} \cong 3.329561 \times 10^{28} \mathrm{~m}^{3} \mathrm{~kg}^{-1} \mathrm{sec}^{-2} \\
& G_{w} \cong 2.909745 \times 10^{22} \mathrm{~m}^{3} \mathrm{~kg}^{-1} \mathrm{sec}^{-2} \\
& G_{N} \cong 6.679855 \times 10^{-11} \mathrm{~m}^{3} \mathrm{~kg}^{-1} \mathrm{sec}^{-2} \\
& \text { (See points-7,8,9,10 of 'Discussion' } \\
& \text { for the supporting relations). }
\end{aligned}
$$

(2) ( $\hbar c)$ can be considered as a compound physical constant,

$$
\hbar c \cong\left(\frac{G_{w}}{G_{s}}\right) G_{e} m_{p} m_{e}
$$

(3) There exists a strong elementary charge $\left(e_{s}\right)$ in such a way that,

$$
\begin{aligned}
& \frac{m_{p}}{m_{e}} \cong\left(\frac{G_{s} m_{p}^{2}}{\hbar c}\right)\left(\frac{G_{e} m_{e}^{2}}{\hbar c}\right) \\
& \left.\cong\left(\frac{e_{s}^{2}}{4 \pi \varepsilon_{0} G_{s} m_{p}^{2}}\right) /\left(\frac{e^{2}}{4 \pi \varepsilon_{0} G_{e} m_{e}^{2}}\right)\right\} \\
& \rightarrow\left\{\begin{array}{l}
\frac{e_{s}^{2}}{e^{2}} \cong\left(\frac{G_{s} m_{p}^{3}}{G_{e} m_{e}^{3}}\right) \cong\left(\frac{G_{s} m_{p}^{2}}{\hbar c}\right)^{2} \cong \frac{1}{\alpha_{s}} \\
\frac{e_{s}}{e} \cong \sqrt{\frac{G_{s} m_{p}^{3}}{G_{e} m_{e}^{3}} \cong\left(\frac{G_{s} m_{p}^{2}}{\hbar c}\right) \cong \sqrt{\frac{1}{\alpha_{s}}}}
\end{array}\right\}
\end{aligned}
$$$$
\text { where, } \alpha_{s} \cong \text { Strong coupling constant }
$$

Based on these relations,

$$
\begin{aligned}
& e_{s} \cong 2.9463591 e \\
& \alpha_{s} \cong 0.1151937 \\
& \frac{1}{\alpha_{s}} \cong 8.681032
\end{aligned}
$$

\section{Understanding proton-neutron stability with} three atomic gravitational constants

In our recently published paper [8], we proposed the following semi empirical relations (4) to (8) for fitting nuclear stability and binding energy.

$$
\begin{aligned}
& s \cong\left\{\left(\frac{e_{s}}{m_{p}}\right) \div\left(\frac{e}{m_{e}}\right)\right\} \cong 0.001605 \\
& \cong \sqrt{\left.\frac{G_{s} m_{p}}{G_{e} m_{e}} \cong \frac{G_{s} m_{p} m_{e}}{\hbar c} \cong \frac{\hbar c}{G_{e} m_{e}^{2}} \cong \frac{G_{s}^{2}}{G_{e} G_{w}} \cong \frac{m_{p}}{M_{w}}\right\}} \\
& \text { where, } M_{w} \cong \sqrt{\hbar c / G_{w}} \cong 584.725 \mathrm{GeV} / c^{2}
\end{aligned}
$$

Nuclear mean beta stability line can be explained with a relation of the form,

$$
\begin{aligned}
& A_{s} \cong Z+N_{s} \\
& \cong 2 Z+s(2 Z)^{2} \cong 2 Z+(4 s) Z^{2} \\
& \cong 2 Z+k Z^{2} \cong Z(2+k Z) \\
& \text { where } k \cong 4 s \cong 0.0064185
\end{aligned}
$$

By considering a factor like $[2 \pm \sqrt{k}]$, likely possible range of $A_{s}$ can be explained with,

$$
\left\{\begin{array}{l}
\left(A_{s}\right)_{\text {lower }} \cong Z(1.92+k Z) \\
\left(A_{S}\right)_{\text {mean }} \cong Z(2.0+k Z) \\
\left(A_{S}\right)_{\text {upper }} \cong Z(2.08+k Z)
\end{array}\right\}
$$

\section{Understanding nuclear binding energy}

For $(Z \approx 3$ to 118$)$, close to beta stability line, nuclear binding energy can be fitted with,

$$
B_{A_{s}} \cong\left\{\left(1-0.00189 \sqrt{Z N_{s}}\right) A_{s}-A_{s}^{1 / 3}-\left(\frac{Z}{N_{s}}\right)\right\} B_{0}
$$

See Figure 1. Dashed red curve plotted with relations (5) and (7) can be compared with the green curve plotted with the standard SEMF. For light, medium and heavy atomic nuclides, fit is reasonable. 
Figure 1: Binding energy per nucleon close to stable mass numbers of $Z=3$ to 118

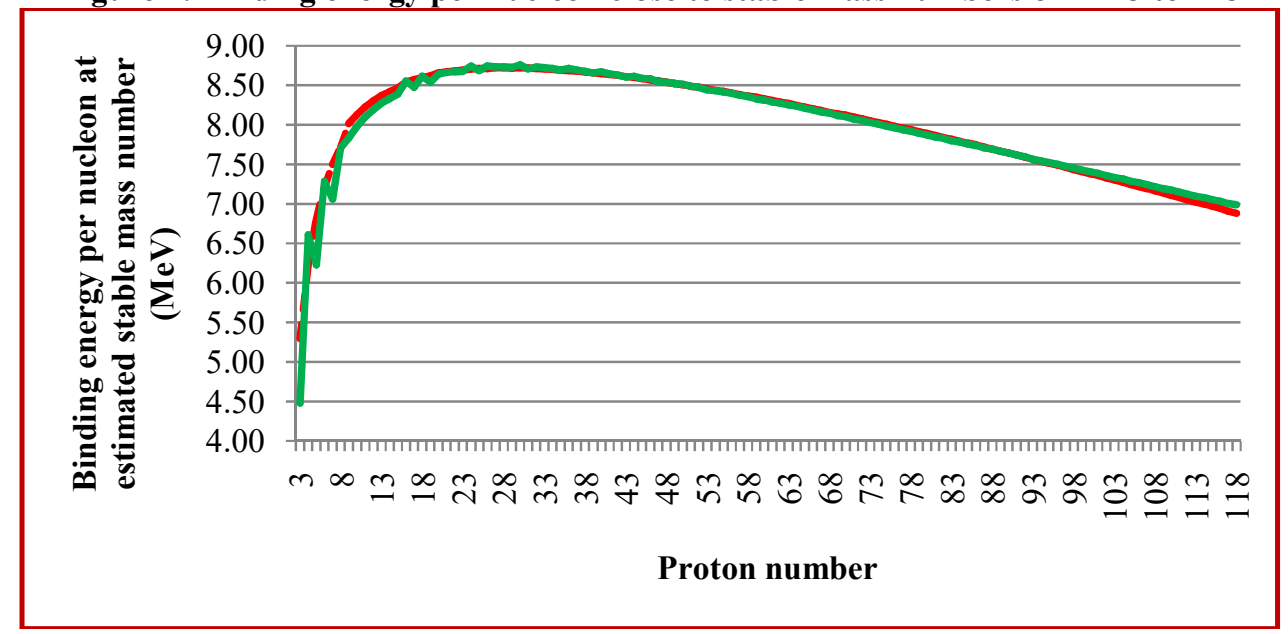

In this section, we try to infer and review relation (7) for its best possible physics back ground. With further study, nuclear stability and binding energy can be understood with Up and Down quarks. We propose that, energy $[8,12,17,18]$ seems to decrease by

(1) Nuclear unit radius can be expressed as, $R_{0} \cong \frac{2 G_{s} m_{p}}{c^{2}} \cong 1.239291 \mathrm{fm}$

(2) $B_{0} \cong \frac{e_{s}^{2}}{4 \pi \varepsilon_{0} R_{0}} \cong \frac{e_{s}^{2}}{8 \pi \varepsilon_{0}\left(G_{s} m_{p} / c^{2}\right)} \cong 10.09 \mathrm{MeV}$ can be considered as the unique binding energy coefficient. With reference to the recommended [21] up quark rest energy of $2.15 \mathrm{MeV}$ and down quark rest energy of $4.7 \mathrm{MeV}$, it is quite interesting to note that, $\frac{\left[\left(2 m_{u}+m_{d}\right) c^{2}+\left(m_{u}+2 m_{d}\right) c^{2}\right]}{2} \cong 10.275 \mathrm{MeV}$

(3) For increasing $(Z, A)$, all nucleons will not involve in nuclear binding energy scheme.

(4) Nucleons that are not involving in nuclear binding energy scheme can be called as 'free nucleons' and can be represented by $A_{f} \cong k_{f} A \sqrt{Z N}$ where the coefficient $k_{f} \cong 0.00189$ can be called as 'Free nucleon number coefficient'. With reference to the semi empirical mass formula, quantitatively, $k_{f} \cong 2\left(a_{c} / a_{a}\right)^{2} \cong 0.0018753$ where $a_{c}=0.71 \mathrm{MeV}$ and $a_{a}=23.21 \mathrm{MeV}$.

(5) Nucleons that involve in nuclear binding energy scheme can be called as 'active nucleons' and can be represented by $A_{a} \cong A-A_{f} \cong A(1-0.00189 \sqrt{Z N})$.
(6) For $Z=11$ to 92 , When $\left(A_{a}-2 Z\right) \cong 0$, corresponding A seems to represent the possible existence of lower stability line.

(7) The ad-hoc coefficient 0.00189 somehow, seems to lie between $\{s \cong 0.0016$ and $k \cong 0.0064\}$. With reference to electromagnetic interaction, we consider, $[k / \ln (30)] \cong 0.00189$ where 30 is a characteristic representation of atomic number below which strength of nuclear binding $[Z / 30]^{\sqrt{k}}\left(1 / \alpha_{s}\right) \cong[Z / 30]^{0.08} \times 8.68$. From $\quad Z=30$ onwards, strength of nuclear binding energy remains constant at $\left(1 / \alpha_{s}\right) \cong 8.68$. See point 4 of 'Discussion'.

(8) Binding energy can be assumed to decrease with increasing radius.

(9) Decreasing proton-neutron ratio seems to play an interesting role in increasing binding energy.

(10) Considering isotopes, stable mass number plays an interesting role in estimating the binding energy of other stable and unstable isotopes in the form of $\left(\left(A_{s}-A\right)^{2} / A_{s}\right)$. It needs further investigation.

Above and below the stable mass numbers, binding energy can be approximated with,

$$
B_{A} \cong\left\{\begin{array}{r}
(1-0.00189 \sqrt{Z N}) A-A^{1 / 3} \\
-\left(\frac{Z}{N}\right)-\frac{\left(A_{s}-A\right)^{2}}{A_{s}}
\end{array}\right\} B_{0}
$$


See Figure 2 for the estimated isotopic binding energy of $\mathrm{Z}=50$. Dotted blue curve plotted with relations (5) and (8) can be compared with the green curve plotted with SEMF.

a) Based on Figures 1 and 2, it is possible to say that, Relations (5), (7) and (8) can also be given some priority in understanding nuclear binding energy scheme.

b) Estimated binding energy can also be compared with spherical relativistic continuum Hartree-Bogoliubov
(RCHB) theory data [20] and Thomas-Fermi model (Table of nuclear masses, nsdssd.lbl.gov, 1994).

c) For $(N<Z)$ and $(N \approx Z)$ estimated binding energy seems to be increasing compared to SEMF estimation.

d) For $\left(A \gg>A_{s}\right)$, estimated binding energy seems to be decreasing compared to SEMF estimation.

Figure 2: Isotopic binding energy of $Z=50$

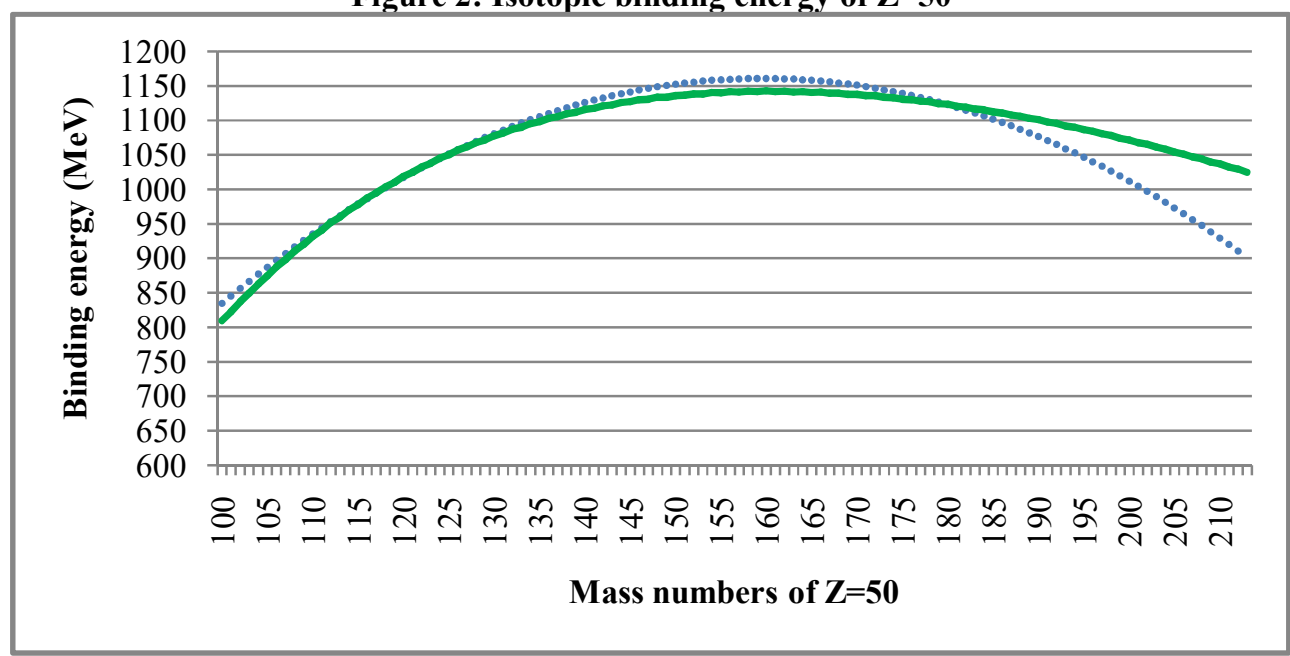

\section{To estimate quark masses}

In our earlier paper [22], we proposed that,

a) Up, Strange and Bottom quarks are in geometric series.

b) Down, Charm and Top quarks are in another geometric series.

We modify these ideas as:

a) Down and Up quarks are ground state particles and their mass ratio is 2 .

b) Strange and Bottom quarks are first generation particles having a geometric series with a geometric ratio

of, $g_{u} \cong \sqrt{m_{p} / m_{e}} \cong \sqrt{1836.152} \cong 42.85$.

c) Charm and Top quarks are second generation particles having another geometric series with a geometric ratio of, $g_{d} \cong(1 / \alpha) \cong 137.036$.

d) These two geometric ratios can be fitted with $\frac{e^{2}}{4 \pi \varepsilon_{0} G_{s} m_{p} m_{e}} \cong 4.547665$.
Considering Up and Down quarks as ground state particles, quark masses [21] can be fitted in the following way.

\section{Step-1: To fit Up quark mass}

$$
\left.\begin{array}{c}
\frac{e^{2}}{4 \pi \varepsilon_{0} G_{s} m_{p} m_{u}} \cong 1 \text { and } \\
\frac{G_{s} m_{p}}{c^{2}} \cong \frac{e^{2}}{4 \pi \varepsilon_{0}\left(m_{u} c^{2}\right)} \\
\rightarrow m_{u} c^{2} \cong 2.324 \mathrm{MeV}
\end{array}\right\}
$$

\section{Step-2: To fit Down quark mass}

$$
\left.\begin{array}{l}
\frac{m_{d} c^{2}}{m_{u} c^{2}} \cong 2 \text { and } \\
m_{d} c^{2} \cong 4.648 \mathrm{MeV}
\end{array}\right\}
$$


Based on the estimated up and down quarks, we noticed that,

$$
\frac{m_{u} c^{2}+m_{d} c^{2}}{m_{n} c^{2}} \cong 0.0074256 \cong \alpha
$$

where $m_{n}=$ average mass of nucleon and $\alpha=$ fine structure constant.

\section{Step-3: To fit Strange and Bottom quark masses}

Considering Strange and Bottom quarks as first generation particles, their masses can be fitted with,

$$
\left.\begin{array}{c}
m_{s} c^{2} \cong \sqrt{\frac{m_{p}}{m_{e}}}\left(m_{u} c^{2}\right) \cong 99.578 \mathrm{MeV} \\
m_{b} c^{2} \cong\left(\sqrt{\frac{m_{p}}{m_{e}}}\right)^{2}\left(m_{u} c^{2}\right) \\
\cong\left(\frac{m_{p}}{m_{e}}\right)\left(m_{u} c^{2}\right) \cong 4266.95 \mathrm{MeV}
\end{array}\right\}
$$

\section{Step-4: To fit Charm and Top quark masses}

Considering Charm and Top quarks as second generation particles, their masses can be fitted with,

$$
\begin{aligned}
& m_{s} c^{2} \cong 2\left(\frac{1}{\alpha}\right)\left(m_{d} c^{2}\right) \cong 1273.806 \mathrm{MeV} \\
& m_{t} c^{2} \cong 2\left(\frac{1}{\alpha}\right)^{2}\left(m_{d} c^{2}\right) \cong 174557.2 \mathrm{MeV}
\end{aligned}
$$

\section{Step-5: To fit the two geometric ratios}

Let,

$$
\frac{e^{2}}{4 \pi \varepsilon_{0} G_{s} m_{p} m_{e}} \cong 4.547665 \cong X
$$

Based on this definition, we noticed that,

$$
\left.\begin{array}{r}
\left(g_{u}, g_{d}\right) \cong\left(\mathrm{e}^{x}-X\right) \mp\left(\frac{\mathrm{e}^{X}}{2}\right) \\
g_{u} \cong\left(\mathrm{e}^{X}-X\right)-\left(\frac{\mathrm{e}^{X}}{2}\right) \cong 42.6582 \\
g_{d} \cong\left(\mathrm{e}^{X}-X\right)+\left(\frac{\mathrm{e}^{X}}{2}\right) \cong 137.069852
\end{array}\right\}
$$

(1) Nuclear binding energy can be understood with a single and unified energy coefficient.

(2) The new numbers $(s$ and $k$ ) seem to play an interesting role in understanding nuclear stability and binding energy. With reference to stable mass number and similar to the famous relation, $Z \cong A /\left(1.98+0.0153 A^{2 / 3}\right)$, proton number can also be estimated with, $Z \cong \frac{A}{1+\sqrt{1+k A}} \cong \frac{\sqrt{k A+1}-1}{k}$.

(3) Considering a term of the form $\left(1-0.00189 \sqrt{Z \sqrt{N N_{s}}}\right)$ or by modifying the terms, $(Z / N)$ and $\left(\left(A_{s}-A\right)^{2} / A_{s}\right)$ binding energy for $\left(A<<A_{S}\right)$ and $\left(A>>A_{S}\right)$ can be understood.

(4) $\mathrm{Z}=(2$ to 118$)$, close to stable mass numbers, binding energy [8] can also be approximated with,

For $\mathrm{Z}<30$ and $A_{s} \cong Z(2+k Z)$,

$\left(B_{A_{s}}\right) \cong\left(\frac{Z}{30}\right)^{0.08}\left\{A_{s}-\left[\left(0.00189 N_{s}^{2}\right)+\frac{1}{2}\right]\right\} 9.16 \mathrm{MeV}$

For $Z \geq 30$ and $A_{s} \cong Z(2+k Z)$,

$\left(B_{A_{s}}\right) \cong\left\{A_{s}-\left[\left(0.00189 N_{s}^{2}\right)+\frac{1}{2}\right]\right\} 9.16 \mathrm{MeV}$

where, $\left\{\begin{array}{l}\frac{e_{s}^{2}}{8 \pi \varepsilon_{0}\left(G_{s} m_{p} / c^{2}\right)}-\left(\frac{e^{2}}{4 \pi \varepsilon_{0} R_{0}}\right) \cong 8.928 \mathrm{MeV} \\ \frac{e_{s}^{2}}{8 \pi \varepsilon_{0}\left(G_{s} m_{p} / c^{2}\right)}-\frac{3}{5}\left(\frac{e^{2}}{4 \pi \varepsilon_{0} R_{0}}\right) \cong 9.395 \mathrm{MeV} \\ \text { and } \frac{8.928+9.395}{2} \cong 9.16 \mathrm{MeV}\end{array}\right\}$

(5) In case of Deuteron, there exists no strong interaction between proton and neutron $[12,15]$.

(6) Nuclear charge radii $[10,18]$ can be expressed as,

$$
R_{(Z, A)} \cong\left\{Z^{1 / 3}+(\sqrt{\mathrm{Z}(A-Z)})^{1 / 3}\right\}\left(\frac{G_{s} m_{p}}{c^{2}}\right)
$$

(7) The following set of four semi empirical relations can be considered as REFERENCE relations [8-18]. They need further investigation.

\section{Discussion}



A) $\frac{m_{p}}{m_{e}} \cong 2 \pi \sqrt{\frac{4 \pi \varepsilon_{0} G_{e} m_{e}^{2}}{e^{2}}}$
B) $\hbar c \cong \frac{\left(G_{e}^{2} G_{N}\right)^{1 / 3} m_{p}^{4}}{m_{e}^{2}}$
C) $\frac{m_{p}}{m_{e}} \cong\left(\frac{G_{s}}{G_{e}^{1 / 3} G_{N}^{2 / 3}}\right)^{1 / 7}$
D) $\frac{G_{w}}{G_{N}} \cong\left(\frac{m_{p}}{m_{e}}\right)^{10}$

\section{Note points:}

a) Relation (22A) is our given definition for $G_{e}$.

b) Inserting (22B) in relation (23), relation (22C) can be obtained.

c) Relation (22d) can be inferred from relation (32).

d) With further study, other possible relations for $G_{e}$ and other set of REFERENCE relations can also be developed.

(8) The fundamental question to be answered is: How to justify the estimated values of $\left(G_{N}, G_{s}, G_{w}\right)$ with respect to defined $G_{e}$ ? With reference to current literature pertaining to gravity, it seems impossible to answer this question. By considering 'quantum gravity', it seems possible to find an answer. We noticed that,

$$
\left.\begin{array}{l}
\left(\frac{G_{s} m_{p}}{c^{2}} / \sqrt{\frac{G_{N} \hbar}{c^{3}}}\right) \cong\left(\frac{m_{p}}{m_{e}}\right)^{6} \\
\left(\frac{G_{s} m_{p}}{c^{2}} / \sqrt{\frac{G_{N} \hbar}{c^{3}}}\right)^{\frac{1}{6}} \cong\left(\frac{m_{p}}{m_{e}}\right)
\end{array}\right\}
$$

In a simplified form,

$$
\frac{G_{s}^{2} m_{p}^{2}}{G_{N} \hbar c} \cong\left(\frac{m_{p}}{m_{e}}\right)^{12}
$$

a) Considering CODATA-2014 recommended big $G \equiv G_{N}$, with relation (24) value of $G_{s}$ can be estimated.

$$
G_{s} \cong\left(\frac{m_{p}^{5}}{m_{e}^{6}}\right) \sqrt{G_{N} \hbar c}
$$

b) By inserting the values of $\left(G_{N}, G_{s}\right)$ in relation (22C), value of $G_{e}$ can be estimated.

$$
G_{e} \cong\left(\frac{m_{e}}{m_{p}}\right)^{21} \frac{G_{s}^{3}}{G_{N}^{2}}
$$

c) By inserting the value of $G_{e}$ in relation (22A), value of $\left(m_{p} / m_{e}\right)$ can be estimated.

$$
\left.\begin{array}{l}
\text { A) }\left(\frac{m_{p}}{m_{e}}\right) \cong 2 \pi \sqrt{\frac{4 \pi \varepsilon_{0} G_{e} m_{e}^{2}}{e^{2}}}(\mathrm{Or}) \\
\text { B) }\left(\frac{m_{p}}{m_{e}}\right) \cong \sqrt[4]{2 \pi \sqrt{\frac{4 \pi \varepsilon_{0} \hbar^{3 / 2} c^{3 / 2}}{e^{2} G_{N}^{1 / 2} m_{e}}}}
\end{array}\right\}
$$

d) Calculating the \%error in the estimated $\left(m_{p} / m_{e}\right)$, error in $G_{N}$ can be reviewed.

e) Based on relation (27A) estimated $\left(m_{p} / m_{e}\right) \cong 1836.549774$ is $216 \mathrm{ppm}$ higher than the recommended $\left(m_{p} / m_{e}\right)$.

f) Based on relation (27B), estimated $\left(m_{p} / m_{e}\right) \cong 1836.251941$ is $54 \mathrm{ppm}$ higher than the recommended $\left(m_{p} / m_{e}\right)$. It is surprising to note that, independent of all the proposed three atomic gravitational constants,

$$
\begin{aligned}
G_{N} & \cong\left(\frac{m_{e}}{m_{p}}\right)^{14}\left(\frac{4 \pi \varepsilon_{0} \hbar c}{e^{2}}\right)^{2}\left(\frac{16 \pi^{4} \hbar c}{m_{p}^{2}}\right) \\
& \cong\left(\frac{m_{e}}{m_{p}}\right)^{14}\left(\frac{1}{\alpha}\right)^{2}\left(\frac{16 \pi^{4} \hbar c}{m_{p}^{2}}\right) \\
& \cong 6.679855429 \times 10^{-11} \mathrm{~m}^{3} \mathrm{~kg}^{-1} \mathrm{sec}^{-2}
\end{aligned}
$$

where, $\alpha \cong$ Fine structure constant

$$
\left.\begin{array}{l}
\text { A) }\left(\frac{m_{p}}{m_{e}}\right) \cong\left\{\frac{4 \pi^{2}}{\alpha} \sqrt{\frac{\hbar c}{G_{N} m_{p}^{2}}}\right\}^{1 / 7} \\
\text { B) } \alpha \cong 4 \pi^{2}\left(\frac{m_{e}}{m_{p}}\right)^{7} \sqrt{\frac{\hbar c}{G_{N} m_{p}^{2}}}
\end{array}\right\}
$$

Based on relation (29), by estimating the error in $\left(\frac{m_{p}}{m_{e}}\right)$ or $\alpha, G_{N}$ value can be reviewed. 
g) By considering $\left(m_{p} / m_{e}\right)$ and $(\alpha)$ as key tools, actual $G_{N}$ value seems to be higher than the CODATA and other big $G$ experimental values. With further study and considering other possible relations for $G_{e}$ and repeating the above steps, $G_{N}$ can be refined.

h) Applying the concept of Penrose model of extraction of black hole energy [23] to a proton, it is also possible to show that [24],

$$
\left(1-\frac{1}{\sqrt{2}}\right) \frac{m_{p}}{m_{e}} \cong\left(\frac{\hbar c}{G_{N} m_{p}^{2}}\right)^{1 / 14}
$$

where,

$$
\begin{aligned}
& \left\{\frac{4 \pi^{2}}{\alpha}\right\}^{\frac{1}{7}} \cong 3.414393 \cong 2+\sqrt{2} \cong\left(1-\frac{1}{\sqrt{2}}\right)^{-1} \\
& G_{N} \cong\left[\left(1-\frac{1}{\sqrt{2}}\right) \frac{m_{p}}{m_{e}}\right]^{-14}\left(\frac{\hbar c}{m_{p}^{2}}\right) \\
& \cong 6.674946866 \times 10^{-11} \mathrm{~m}^{3} \mathrm{~kg}^{-1} \mathrm{sec}^{-2}
\end{aligned}
$$

This estimated value is $130 \mathrm{ppm}$ higher than the CODATA-2014 recommended value. At the same time, it seems to lie in between HUST-AAF-2018 result of $6.674484 \times 10^{-11} \mathrm{~m}^{3} \mathrm{~kg}^{-1} \mathrm{sec}^{-2}$ and BIPM2014 result of $6.67554 \times 10^{-11} \mathrm{~m}^{3} \mathrm{~kg}^{-1} \mathrm{sec}^{-2}$. See Table 1 for the historical results [25-32] of $G_{N}$.

Table 1: Various experimental values of $G_{N}$

\begin{tabular}{|c|c|}
\hline Experiment/Year & $G_{N} / 10^{-11} \mathrm{~m}^{3} \mathrm{~kg}^{-1} \mathrm{sec}^{-2}$ \\
\hline NIST-1982 & 6.67248 \\
\hline TR\&D-1996 & 6.6729 \\
\hline LANL-1997 & 6.67398 \\
\hline UWash-2000 & 6.674255 \\
\hline BIPM-2001 & 6.67559 \\
\hline UWup-2002 & 6.67422 \\
\hline MSL-2003 & 6.67387 \\
\hline HUST-2005 & 6.67222 \\
\hline UZur-2006 & 6.67425 \\
\hline HUST-2009 & 6.67349 \\
\hline JILA-2010 & 6.6726 \\
\hline BIPM-2014 & $\mathbf{6 . 6 7 5 5 4}$ \\
\hline LENS-2014 & 6.67191 \\
\hline UCI-2014 & 6.67435 \\
\hline HUST-TOS-2018 & 6.674184 \\
\hline HUST-AAF-2018 & $\mathbf{6 . 6 7 4 4 8 4}$ \\
\hline
\end{tabular}

(9) Fermi's weak coupling constant is one of the most critical and complicated nuclear physical constant. It can be approximated as,

$$
\left.\begin{array}{rl}
G_{F} & \cong\left(\frac{m_{e}}{m_{p}}\right)^{2} \hbar c R_{0}^{2} \\
& \cong\left[\left(G_{e}^{2} G_{N}\right)^{1 / 3} m_{p}^{2}\right]\left(\frac{2 G_{s} m_{p}}{c^{2}}\right)^{2} \\
& \cong\left(\frac{m_{p}}{m_{e}}\right)^{10} \frac{4 G_{N} \hbar^{2}}{c^{2}} \cong \frac{4 G_{w} \hbar^{2}}{c^{2}} \\
& \cong 1.44021048 \times 10^{-62} \mathrm{~J} \cdot \mathrm{m}^{3}
\end{array}\right\}
$$

This estimated value is $3036 \mathrm{ppm}$ higher than the recommended value, $G_{F} \cong 1.435850984 \times 10^{-62} \mathrm{~J} . \mathrm{m}^{3}$ where $G_{F} /(\hbar c)^{3} \cong 1.1663787 \times 10^{-5} \mathrm{GeV}^{-2}[28]$.

(10) The basic question to be answered is: How do the invoked four gravitational constants address the issues pertaining to vector forces of electromagnetism, tensor forces of gravity and vector-axial vector forces of weak interaction and gluons of strong interaction? It needs further study with respect to the four gravitational constants and the compound $(\hbar)$. In this context, we could notice that, 'Range of four interactions' can be expressed by a model relation of the form [24],

$$
r_{x} \approx\left(M_{x} / m_{p}\right) \sqrt{G_{x} \hbar / c^{3}}
$$

where $M_{x}, G_{x}, m_{p}$ represent the characteristic mass of interaction, characteristic gravitational constant and proton mass respectively. As most of the atomic matter is characterised by protons, this relation can be given some consideration.

a) Strong interaction range,

$$
r_{s} \approx \sqrt{G_{s} \hbar / c^{3}} \approx 0.361 \times 10^{-15} \mathrm{~m}
$$

b) Weak interaction range,

$$
\begin{aligned}
r_{w} & \approx(80400 \mathrm{MeV} / 938.272 \mathrm{MeV}) \sqrt{G_{w} \hbar / c^{3}} \\
& \approx 2.892 \times 10^{-17} \mathrm{~m}
\end{aligned}
$$

c) Electromagnetic interaction range at atomic level, 


$$
\begin{aligned}
r_{e m} & \approx(931.5 \mathrm{MeV} / 938.272 \mathrm{MeV}) \sqrt{G_{e} \hbar / c^{3}} \\
& \approx 9.57 \times 10^{-12} \mathrm{~m} \approx 9.57 \mathrm{pm}
\end{aligned}
$$

d) Gravitational interaction range for Sun,

$$
\begin{aligned}
r_{\text {sun }} & \approx\left(2.0 \times 10^{30} \mathrm{~kg} / 1.672 \times 10^{-27} \mathrm{~kg}\right) \sqrt{G_{N} \hbar / c^{3}} \\
& \approx 1.933 \times 10^{22} \mathrm{~m}
\end{aligned}
$$

\section{Conclusion}

Understanding nuclear binding energy with single energy coefficient in terms of fundamental interactions is a very challenging task. In this context, we tried our level best in presenting a very simple and effective semi empirical formula with one unique energy coefficient. It needs further investigation.

Even though derivational procedure is missing, consequences of the proposed four reference relations $(22 \mathrm{~A}, 22 \mathrm{~B}, 22 \mathrm{C}$ and 22D) seem to be quite interesting. By implementing the four gravitational constants in String theory models [33], it may be possible to explore the hidden unified physics connected with compound $(\hbar c)$, different forms of fundamental forces and their interaction ranges. Finally, gap between nuclear scale and Planck scale can be understood with proper physics independent of large numbers. Proceeding further, theoretical value of $G_{N}$ can be defined as a standard reference for future nuclear, atomic and gravitational experiments [34].

\section{References}

[1] K. Tennakone, Electron, muon, proton, and strong gravity. Phys. Rev. D, 10, 1722 (1974)

[2] C. Sivaram and K. Sinha, Strong gravity, black holes, and hadrons. Physical Review D., 16(6), 1975-1978 (1977)

[3] De Sabbata V and M. Gasperini. Strong gravity and weak interactions. Gen. Relat. Gravit. 10, 9, 731-741, (1979)

[4] Salam A, Sivaram C. Strong Gravity Approach to QCD and Confinement. Mod. Phys. Lett., v. A8(4), 321-326 (1993)

[5] Roberto Onofrio. On weak interactions as shortdistance manifestations of gravity. Modern Physics Letters A 28, 1350022 (2013)

[6] Seshavatharam UVS, Lakshminarayana S. To confirm the existence of atomic gravitational constant. Hadronic Journal.34(4):379 (2011)
[7] Seshavatharam UVS, Lakshminarayana S. Molar electron mass and the basics of TOE. Journal of Nuclear and Particle Physics. 2(6): 132 -141 (2012)

[8] Seshavatharam UVS and Lakshminarayana S. On the Role of Squared Neutron Number in Reducing Nuclear Binding Energy in the Light of Electromagnetic, Weak and Nuclear Gravitational Constants - A Review. Asian Journal of Research and Reviews in Physics, 2(3): 1-22, (2019)

[9] Seshavatharam UVS et al. Understanding the constructional features of materialistic atoms in the light of strong nuclear gravitational coupling. Materials Today: 3/10PB, Proceedings 3, 39763981 (2016)

[10] Seshavatharam UVS and Lakshminarayana S. Towards a workable model of final unification. International Journal of Mathematics and Physics 7(1), 117-130 (2016)

[11] Seshavatharam UVS and Lakshminarayana S. Understanding the basics of final unification with three gravitational constants associated with nuclear, electromagnetic and gravitational interactions. Journal of Nuclear Physics, Material Sciences, Radiation and Applications 4(1),1-19 (2017)

[12] Seshavatharam UVS and Lakshminarayana S. On the role of 'reciprocal' of the strong coupling constant in nuclear structure. Journal of Nuclear Sciences, 4( 2), 31-44 (2017).

[13] Seshavatharam UVS and Lakshminarayana S. Fermi scale applications of strong (nuclear) gravity-1. Proceedings of the DAE Symp. on Nucl. Phys. 63, 72-73. (2018)

[14] Seshavatharam UVS and Lakshminarayana S. A virtual model of microscopic quantum gravity, Prespacetime Journal, 9(1), 58-82 (2018).

[15] Seshavatharam UVS and Lakshminarayana S. On the role of four gravitational constants in nuclear structure. Mapana Journal of Sciences, 18(1), 2145 (2019)

[16] Seshavatharam UVS and Lakshminarayana S. On the Role of Large Nuclear Gravity in Understanding Strong Coupling Constant, Nuclear Stability Range, Binding Energy of Isotopes and Magic proton numbers - A Critical Review. J. Nucl. Phys. Mat. Sci. Rad. A. 6(2), 142-160 (2019)

[17]Cht. Mavrodiev S, Deliyergiyev MA. Modification of the nuclear landscape in the inverse problem framework using the generalized Bethe-Weizsäcker mass formula. Int. J. Mod. Phys. E27: 1850015 (2018)

[18] Xiaa XW, et al. The limits of the nuclear landscape explored by the relativistic continuum Hatree-Bogoliubov theory. Atomic Data and Nuclear Data Tables. 121-122: 1-215 (2018) 
[19] Ghahramany $\mathrm{N}$ et al. New scheme of nuclide and nuclear binding energy from quark-like model. Iranian Journal of Science \& Technology. A3: 201-208 (2011)

[20] Ghahramany $\mathrm{N}$ et al. New approach to nuclear binding energy in integrated nuclear model. Journal of Theoretical and Applied Physics. 6:3 (2012)

[21] M. Tanabashi et al. (Particle Data Group), Phys. Rev. D 98, 030001 (2018)

[22] Seshavatharam UVS and Lakshminarayana S. Super symmetry in strong and weak interactions, Int. J. Mod. Phys. E 19 (2), 263-280 (2010)

[23]R. Penrose and R. M. Floyd, Extraction of Rotational Energy from a Black Hole. Nature Physical Science 229, 177 (1971)

[24] Seshavatharam UVS and Lakshminarayana S. On the role of Newtonian gravitational constant in estimating Proton-Electron mass ratio and baryon mass spectrum. International Journal of Innovative Studies in Sciences Engineering Technology. Vol 5, Issue 10, 18-21 (2019)

[25]B. Canuel et al. Exploring gravity with the MIGA large scale atom interferometer. Science reports, 8:14064 (2018)

[26]Christos Merkatas et al. Shades of dark uncertainty and consensus value for the Newtonian constant of gravitation. arXiv:1905.09551v1 (2019)

[27] Li, Qing et al. Measurements of the gravitational constant using two independent methods. Nature 560, 582-588 (2018)

[28] P. J. Mohr, D. B. Newell, and B. N. Taylor, CODATA recommended values of the fundamental constants: Rev. Mod. Phys. 88, 035009 (2014)

[29]G. Rosi, F. Sorrentino, L. Cacciapuoti, M. Prevedelli, and G. M. Tino. Precision measurement of the Newtonian gravitational constant using cold atoms, Nature 510 (7506), 518-521 (2014)

[30] S. Schlamminger and R.D. Newman. Recent measurements of the gravitational constant as a function of time. Phys. Rev. D 91, 121101 (2015)

[31]C. Rothleitner and S. Schlamminger. Measurements of the Newtonian constant of gravitation, G. Rev. Sci. Instrum. 88, 111101 (2017)

[32] G. Rosi, Challenging the big $G$ measurement with atoms and light. J. Phys. B: At., Mol. Opt. Phys. 49(20), 202002 (2016)

[33] Joseph Polchinski. String theory to the rescue. arXiv:1512.02477v5 [hep-th] 16 Dec 2015

[34] Seshavatharam UVS and Lakshminarayana S. To correlate big $\mathrm{G}$ experiments and other nuclear experiments via three atomic gravitational constants. Dec.20-21,ICAPPM-2019, Hyderabad, India. (To be appeared in IOP Physics conference series). 